M.G. Burton, R. Jayawardhana \& T.L. Bourke, eds.

\title{
Probing AU-scale Structure using Spectro-astrometry
}

Michihiro Takami

Department of Physical Sciences, University of Hertfordshire, College Lane, Hatfield, Herts, AL10 9AB, UK

Jeremy Bailey

Anglo-Australian Observatory, PO Box 296, Epping, NSW 1710, Australia

Antonio Chrysostomou

Department of Physical Sciences, University of Hertfordshire, College Lane, Hatfield, Herts, AL10 9AB, UK

Motohide Tamura

National Astronomical Observatory of Japan, Osawa, Mitaka, Tokyo 181-8588, Japan

Hiroshi Terada

Subaru Telescope, 650 North A'ohoku Place, Hilo, Hawaii 96720, USA

\begin{abstract}
The circumstellar environment within $10 \mathrm{AU}$ of young stars are of particular interest for star and planet formation. Unfortunately, present imaging facilities such as the Hubble Space Telescope or adaptive optics on $10-\mathrm{m}$ telescopes cannot resolve this region. We have proved that "spectro-astrometry" is a powerful technique for discovering premain sequence binaries, determining kinematics of outflows and providing evidence for gaps in circumstellar disks - all down to AU scales. In this paper, we summarise our progress to date.
\end{abstract}

\section{Introduction}

The circumstellar environment within $10 \mathrm{AU}$ of young stars are of particular interest for star and planet formation. In this region, the star accretes material from the circumstellar disk, and drives an energetic jet/wind (see, e.g., Najita et al. 2000; Shu et al. 2000, Königl \& Pudritz 2000). In the circumstellar disk, planets may be growing and tidally interact with the circumstellar environment (see e.g., Nelson et al. 2000). This region also includes a stellar companion in a significant number of objects, and the population, mass distribution, and orbital parameters of these companions could give useful constraints on the understanding of binary formation (see e.g., Mathieu et al. 2000). However, even the Hubble Space Telescope or adaptive optics on 10-m telescopes cannot 

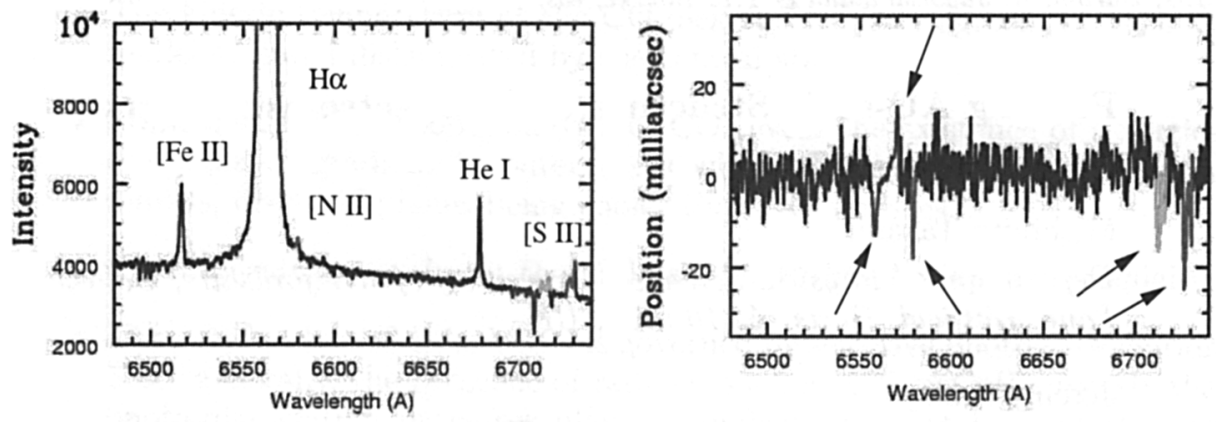

Figure 1. Intensity (left) and position (right) spectra obtained in a $\mathrm{T}$ Tauri star. The position spectrum shows displacement in blueshifted and redshifted wings in $\mathrm{H} \alpha$ emission, and [SII] 6716/6731 $\AA$ and [NII] $6584 \AA$ emission.

resolve this region: a spatial scale of $10 \mathrm{AU}$ corresponds to 0 ".07 in the nearest star forming regions (SFRs), which is comparable to the angular resolutions of these facilities. Observational studies in this region have thus been carried out by photometry and spectroscopy without any direct spatial information, and optical-to-radio interferometry in a limited number of objects.

To obtain spatial information well below these resolutions, we have begun "spectro-astrometric" observations of pre-main sequence (PMS) stars. Through the observations to date, we have proved that the technique is a powerful probe for discovering binaries, determining kinematics of outflows and providing evidence for gaps in circumstellar disks - all down to AU scales, as described below.

\section{Technique of Spectro-astrometry}

Spectro-astrometry requires no special instrumentation. A standard long-slit spectrum obtained with a configuration that gives good sampling of the seeing profile is analysed by centroiding or profile-fitting in the spatial direction. This provides the position of the source at each wavelength (see Fig. 1). The resulting "position spectrum" will show structure for any spectral feature that is displaced from the centroid of the source, e.g., an emission line arising in a binary companion, or in outflowing gas, or any other structure which is not perfectly symmetric about the continuum source. Any instrumental effects (e.g., distortion in the spectrograph camera, imperfect flat fielding) can be removed by subtracting two position spectra taken at anti-parallel position angles (e.g., 0 and $180^{\circ} ; 90$ and $270^{\circ}$ ). This is because displacement due to real structure in the source will have opposite signs, whereas instrumental effects will remain constant. By combining the position spectra observed at orthogonal position angles, we can obtain the displacement in the two dimensions (see Fig. 2).

The measured positional accuracy depends on the seeing size and the photon number at each wavelength. Each photon should be within a distance of the order of the seeing size from the actual position of the source, and this 

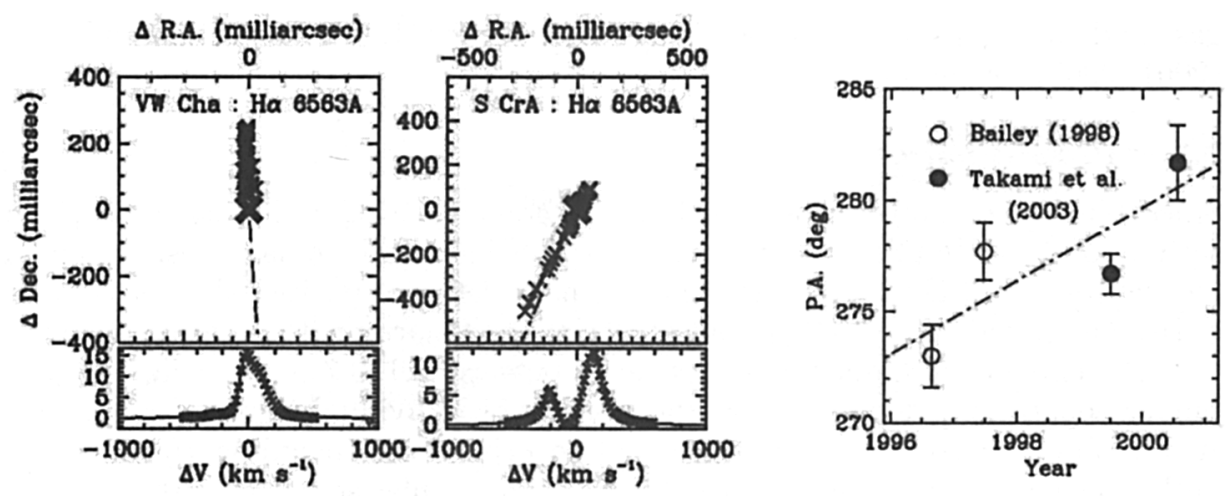

Figure 2. (left) Positional displacement of $\mathrm{H} \alpha$ emission in known binaries. Small crosses indicate the displacement in the two-dimensional sky, and each cross corresponds to a velocity component shown in the line profile. A large cross at the centre indicates the position of the continuum emission. A dot-dashed line indicates the direction of the secondary star. (right) Position angles measured in T CrA. A dotdashed line shows the regression line with an inclination of $1.65^{\circ} \mathrm{yr}^{-1}$

positional uncertainty is reduced by a factor of $N^{1 / 2}$ by observing $N$ photons. Consequently, the detection limit is described as follows (see Takami, Bailey, \& Chrysostomou 2003):

$$
\sigma(\text { milliarcsecond })=\text { const } \cdot \operatorname{FWHM}(\text { milliarcsecond }) \cdot N^{-1 / 2},
$$

where FWHM is the full width half maximum of the seeing. The constant value was measured to be $0.5 \pm 0.2$, in agreement with that estimated using a Gaussian seeing profile (0.4). This implies that a combination of an arcsecond seeing and $3 \times 10^{4}$ photons provides an accuracy of $\sim 2$ milliarcsecond.

The concept of the technique was introduced by Beckers (1982) and Christy et al. (1983) as "differential speckle interferometry" or "chromatic position difference". Solf \& Böhm (1993) and Hirth, Mundt, \& Solf $(1994,1997)$ carried out experiments with a standard common-user spectrograph, measuring the displacement in forbidden line emission of $\mathrm{T}$ Tauri stars down to $\sim 0$ ".1 scales. Bailey (1998a) extended their method, and show that the technique allows us to study the spatial structure of emission/absorption features down to milliarcsecond scales.

\section{Results at Optical Wavelengths}

To date, we have observed about 40 PMS stars at the AAT 3.9-m using the RGO spectrograph. Most of the targets are located in the nearest southern SFRs (130200 pc) including Lupus, Camaeleon, Corona-Australis, Ophiuchus, and Upper Scorpius. The spectra for each object cover $\mathrm{H} \alpha$, HeI, FeII, [SII], and [NII] at $6500-6750 \AA$, and also [OI] $6300 \AA$ for a few objects, with a spectral resolution of $R \sim 7000$. In this section, we summarise the results for PMS binaries, outflowing 
gas, and disk gaps/holes, respectively. See Bailey (1998b) and Takami et al. (2001, 2003) for details.

\subsection{PMS Binaries}

Since binary components usually have different spectra with each other, the seeing position in emission features are displaced along the binary position angle (see left plots in Fig. 2). In particular, we have detected positional displacement of $\mathrm{H} \alpha$ emission in almost all (10 out of 12) known binaries with separations down to 0".1. The observed angular scales varies between $0.1-0.7$ times as large as the binary separation, depending on the spectra of individual binary component. A few objects also exhibit displacement in other lines including HeI $6678 \AA$, FeII 6456/6516 $\AA$, and [SII] 6716/6731 $\AA$. Their angular scales are 0.02-0.05 per binary separation, much smaller than that in $\mathrm{H} \alpha$ emission.

The measured angular scales in $\mathrm{H} \alpha$ and positional accuracy achieved (down to 1 milliarcsecond) suggest that the technique is capable of detecting binaries with separations down to $\sim 10$ milliarcsecond at the $3-\sigma$ level. This detection limit is comparable to that of lunar occultations (see e.g., Simon et al. 1995), although we can easily achieve better specto-astrometric accuracies by integrating for longer times. In addition, we can apply the technique for objects with any coordinate and binary position angles. Its high capability of detecting binaries is proved by the detection of a few binaries, which have not been detected by the infrared speckle technique (T CrA, MWC 300, and possibly R CrA - see Bailey et al 1998; Takami et al. 2003).

The technique also allows us to measure the angular motion of binaries. The right plot in Fig. 2 shows the the measured position angle in $\mathrm{T}$ CrA, which increases with time by $\sim 2^{\circ} \mathrm{yr}^{-1}$. Together with a lower limit of binary separation measured from our results (140 milliarcsecond), we derive a lower limit of the stellar mass of $0.2 \mathrm{M}_{\odot}$, consistent with the mass of a $\mathrm{T}$ Tauri star.

\subsection{Jets/winds}

A few active PMS stars show displacement of $\mathrm{H} \alpha$ emission due to outflowing gas. Two of them (Z CMa, AS 353A) exhibit displacement only in a blueshifted component, while the remaining two (RU Lup and CS Cha) exhibit a bipolar structure (see Fig. 3 for the results in RU Lup). The direction and velocity of the displacement coincide with a forbidden line jet in three of them ( $\mathrm{Z} \mathrm{CMa}, \mathrm{AS}$ 353A, RU Lup), indicating that the displacement is due to the base of the jet. The presence of a forbidden line jet has not been reported in CS Cha, although $\mathrm{H} \alpha$ emission in this object is displaced perpendicular to the optical continuum polarization, which is very often perpendicular to the jet in young stellar objects: thus, we conclude that the observed displacement is also due to outflowing gas.

The results in RU Lup and CS Cha are intriguing, since the observed spatial scale of the $\mathrm{H} \alpha$ outflows (1-5 AU, corresponding to a few tens of milliarcseconds) are much smaller than the resolutions of the Hubble Space Telescope or adaptive optics on 10-m telescopes. The measured spatial scale, velocity, and $\mathrm{H} \alpha$ luminosity suggest the mean hydrogen number density of $\gtrsim 10^{7} \mathrm{~cm}^{-3}$ in these outflows (see Takami et al. 2003 for details). The following trends suggest that these outflows are not heated by shocks in the internal working surfaces, which are responsible for the heating in the jet in the outer ( $>10 \mathrm{AU})$ region. 

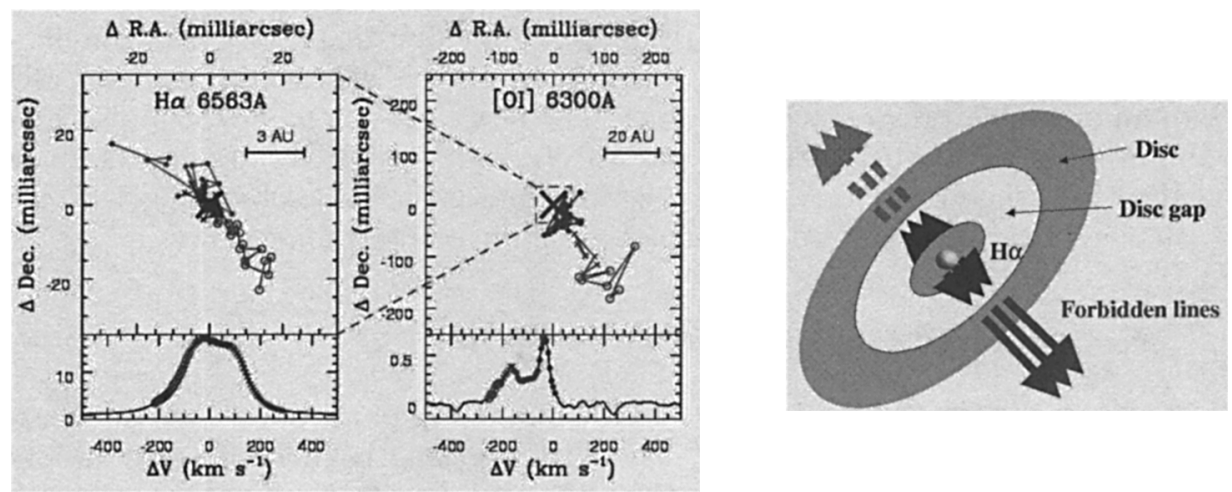

Figure 3. (left) Positional displacement and line profiles for $\mathrm{H} \alpha$ and [OI] outflows in RU Lup (Takami et al. 2001). (right) Schematic view of the $\mathrm{H} \alpha$ and forbidden line outflows, circumstellar disk, and disk gap in the same object.

First, the spatial scales of these $\mathrm{H} \alpha$ outflows are much smaller than the distances between internal working surfaces of the jet associated with PMS stars $(z 100$ AU). Secondly, their line profiles do not exhibit a peak at the velocity of the jet, indicating that the emission line region is continuously distributed from the accelerating region. These AU-scale jets may be heated in the same way as the magnetospheric accretion columns, i.e., by X-ray or MHD waves.

In addition to $\mathrm{H} \alpha$, displacement is also observed in forbidden line outflows in some objects. Their line profiles often show two blueshifted components (see e.g., Fig. 2), and the high-velocity component (HVC) shows a larger displacement than the low velocity component (LVC), consistent with the previous observations with lower positional accuracies (e.g., Hirth et al. 1997). In RU Lup, the displacement in the HVC increases with the velocity, while it remains constant in the LVC, exhibiting different internal kinematics between the two components (see Takami et al. 2001 for details).

\subsection{Disk gaps/holes}

Forbidden line outflows in PMS stars usually exhibit only blueshifted components with a typical spatial scale of more than 30 AU (see Hirth et al. 1997). The absence of redshifted components is attributed to the obscuration of the counterflow by a circumstellar disk. In contrast, $\mathrm{H} \alpha$ emission in RU Lup and CS Cha exhibit a bipolar structure at a few AU. Such results can be explained if the AU-sized disk gap/hole allows redshifted $\mathrm{H} \alpha$ to be seen (see right figure in Fig. 3). Indeed, the spectral energy distribution in these objects exhibit a dip at a mid-infrared wavelength (see Takami et al. 2001; Gauvin \& Strom 1992), consistent with the lack of dusty material at AU scales.

Model calculations have shown that such an AU-sized gap/hole could be induced by tidal interaction with a stellar companion (see e.g., Lubow \& Artimowicz 2000) or a young planet (see e.g., Nelson et al. 2000). The presence of such gaps/holes in young circumstellar disks have been suggested by detailed 
analysis of the spectral energy distribution, although it does not give a convincing proof for its presence (see e.g., Chiang \& Goldreich 1999). Our results in RU Lup and CS Cha provide evidence for the presence of such a gap/hole in young circumstellar disks. In particular, our spectro-astrometric results do not show evidence for the presence of a stellar companion in the same objects, consistent with the scenario that these gaps/holes are induced by young planets.

\section{Near-infrared Observations at SUBARU}

In addition to optical wavelengths, we are extending our studies into the nearinfrared (NIR) at SUBARU 8.2-m. The NIR wavelengths include a rich variety of lines including molecular bands $\left(\mathrm{H}_{2}, \mathrm{CO}\right)$, and also atomic lines with low-tohigh excitation ([FeII], HeI). These lines allow us to study the physical conditions of circumstellar environments in great detail. Furthermore, we expect higher positional accuracy due to the large diameter of SUBARU and low extiction at the observed wavelengths.

The results are still preliminary, although these are promising. We detected a known binary in HeI $1.08 \mu \mathrm{m}, \operatorname{Pa} \gamma 1.09 \mu \mathrm{m}$, and $\mathrm{Pa} \beta 1.28 \mu \mathrm{m}$, and succeeded in measuring its angular motion. In addition, we detected the displacement in $\mathrm{H}_{2} 2.12 \mu \mathrm{m}$ emission in a $\mathrm{T}$ Tauri star, proving that the emission in this object originates from the outflowing gas close to the star. The first results obtained at near-infrared wavelengths were published in Takami et al. (2002), and the remaining results will appear in future papers.

\section{References}

Bailey, J. A., 1998a, Proc. SPIE, 3355, 932

Bailey, J., 1998b, MNRAS, 301, 161

Beckers, J. M. 1981, Lowell Observatory Bulletin, 9, 165

Chiang, E. I. \& Goldreich, P. 1999, ApJ, 519, 279

Christy, J. W., Wellnitz, D. D., \& Currie, D. G. 1983, Lowell Observatory Bulletin, 167, 28

Gauvin, L. S. \& Strom, K. M. 1992, ApJ, 385, 217

Hirth, G. A., Mundt, R., \& Solf, J. 1994, A\&A, 285, 929

Hirth, G. A., Mundt, R., \& Solf, J., 1997, A\&AS, 126, 437

Königl, A., Pudritz, R. E., 2000, Protostars and Planets IV, 759

Mathieu, R. D., Ghez, A. M. et al. 2000, Protostars and Planets IV, 703

Najita, J. R., Edwards, S. et al. 2000, Protostars and Planets IV, 457

Nelson, R. P., Papaloizou, J. C. B. et al. 2000, MNRAS, 318, 18

Shu, F. H., Najita, J. R. et al. 2000, Protostars and Planets IV, 789

Simon, M., Ghez A. M. et al. 1995, ApJ, 443, 625

Solf, J. \& Böhm, K. H. 1993, ApJ, 410, L31

Takami, M., Bailey, J., Chrysostomou, A. 2003, A\&A, 397, 675

Takami, M., Bailey, J., Gledhill, T. M. et al. 2001, MNRAS, 323, 177

Takami, M., Chrysostomou, A., Bailey, J. et al. 2002, ApJ, 568, L53 


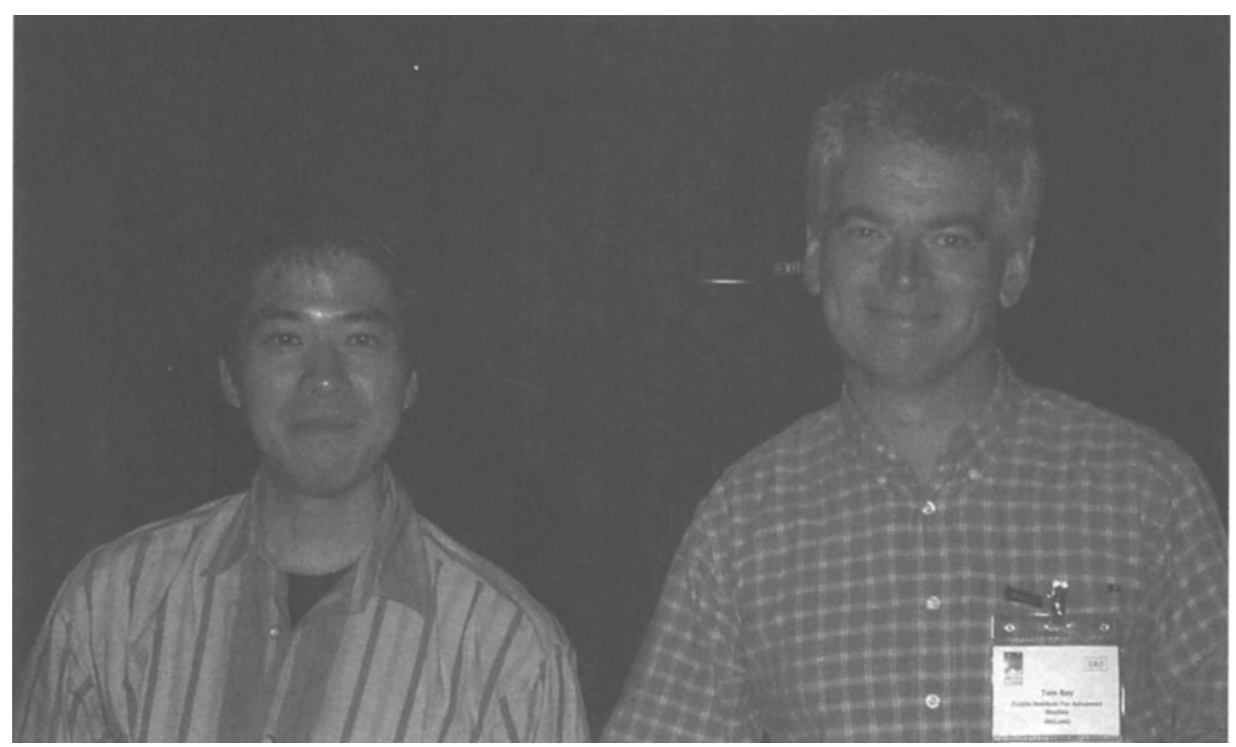

Michihiro Takami and Tom Ray 


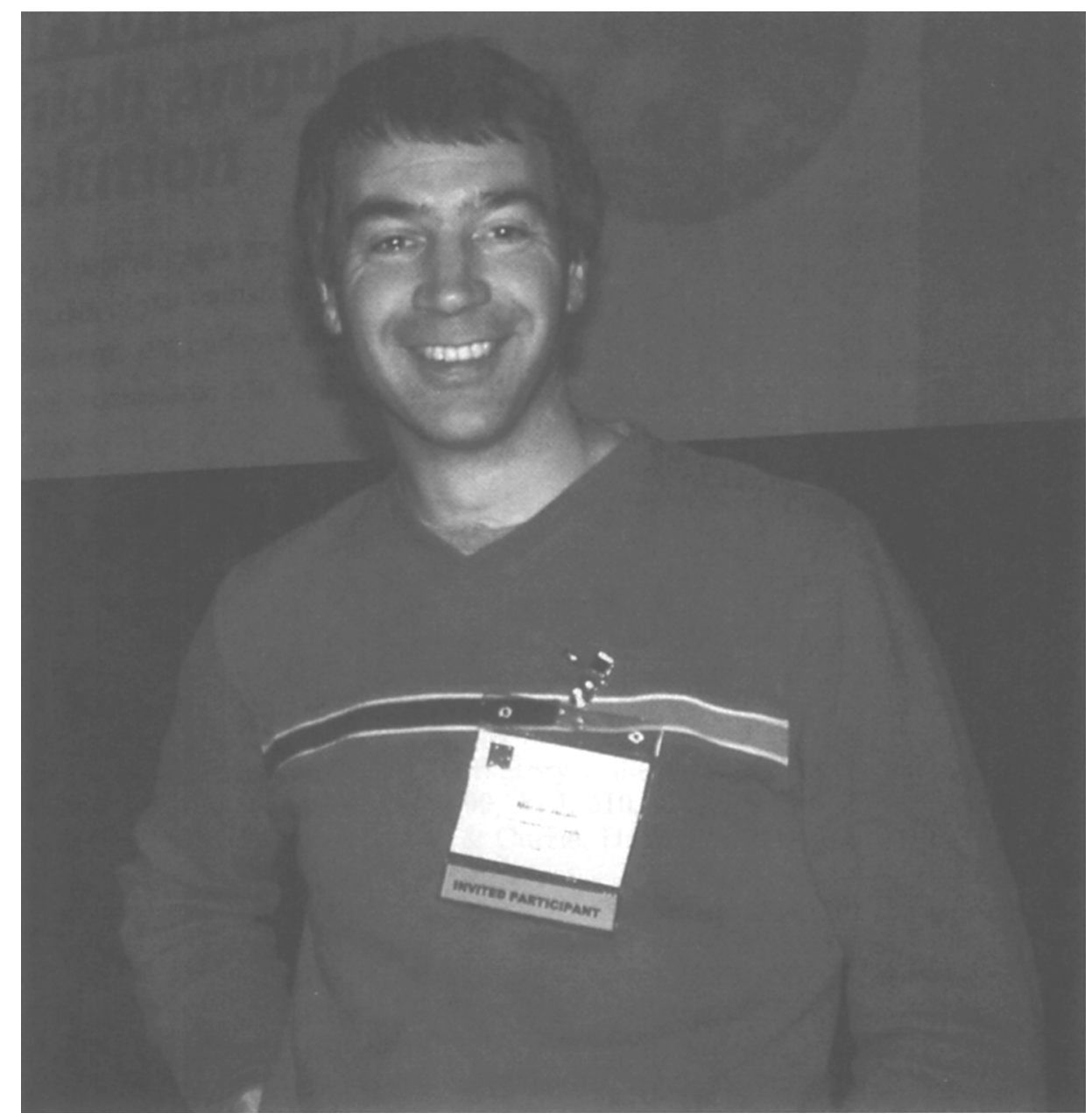

Melvin Hoare 